\title{
A RIGIDITY PROPERTY FOR THE SET OF ALL CHARACTERS INDUCED BY VALUATIONS
}

BY

\author{
ROBERT BIERI AND JOHN R. J. GROVES
}

\begin{abstract}
If $K$ is a field and $G$ a finitely generated multiplicative subgroup of $K$ then every real valuation on $K$ induces a character $G \rightarrow \mathbf{R}$. It is known that the set $\Delta(G) \subseteq \mathbf{R}^{n}$ of all characters induced by valuations is polyhedral. We prove that $\Delta(G)$ satisfies a certain rigidity property and apply this to give a new and conceptual proof of the Brewster-Roseblade result [4] on the group of automorphisms of $K$ stabilizing $G$.
\end{abstract}

\section{Introduction.}

1.1. Let $K$ be a field and $k \subseteq K$ a subfield. By a real valuation on $K$ over $k$ we mean a homomorphism $w: K^{\times} \rightarrow \mathbf{R}$ of the multiplicative group of $K$ into the additive group of the reals which is trivial on $k^{\times}$and satisfies $w(a+b) \geqslant$ $\min \{w(a), w(b)\}\}$ for all $a, b \in K^{\times}$with $a+b \neq 0$. Throughout the paper $G$ shall denote a finitely generated multiplicative subgroup of $K$. Then $w$ induces a character $\chi=\left.w\right|_{G}: G \rightarrow \mathbf{R}$ on $G$ and so, following G. M. Bergman [1], we consider the set $\Delta(G)$ of all characters of $G$ induced by real valuations on $K$ over $k$. Thus $\Delta(G)$ is a subset of the real vector space $G^{*}=\operatorname{Hom}(G, \mathbf{R}) \cong \mathbf{R}^{n}$, where $n=\operatorname{rk} G$ is the torsion free rank of $G$. Since valuations on the subfield $k(G)$ can always be extended to the field $K, \Delta(G)$ depends only on $k(G)$ and not on $K$.

In [2] we have shown that the subset $\Delta(G) \subseteq \mathbf{R}^{n}$ has some special geometric features. In particular, we established Bergman's conjecture that $\Delta(G)$ is a rational spherical polyhedron, i.e., a finite union of finite intersections of closed half spaces given by inequalities with integer coefficients. Here we continue the investigation of the geometry of $\Delta(G)$ by showing that it decomposes into a cartesian product $\Delta(G)=\mathbf{R}^{m} \times \Delta_{1}$ of an affine space $\mathbf{R}^{m}$ and a polyhedron $\Delta_{1} \subseteq \mathbf{R}^{n-m}$ satisfying a certain rigidity property.

1.2. We sketch our main result. In the situation above the group $G$ contains two interesting subgroups $A \leqslant B \leqslant G$, which are in some sense dual to one another. On the one hand we have the unique maximal subgroup $A$ subject to the condition that $k(A)$ is algebraic over $k$ (thus $A$ only consists of all elements of $G$ which are algebraic over $k$ ); and on the other hand $G$ contains a unique minimal subgroup $B$ subject to the condition that $k(G)$ is purely transcendental over $k(B)$ (equivalently, the number of generators of $G / B$ coincides with $\operatorname{tr} \operatorname{deg}_{k(B)} k(G)$, the transcendence degree of $k(G)$ over $k(B))$. Note that the factors $G / B$ and $B / A$ are torsion free.

Received by the editors November $1,1983$.

1980 Mathematics Subject Classification. Primary 13A18, 12 F99.

C1986 American Mathematical Society $0002-9947 / 86 \$ 1.00+\$ .25$ per page 
Any subgroup $H \leqslant G$ gives rise to a short exact sequence of $\mathbf{R}$-vector spaces $(G / H)^{*} \neg G^{*} \rightarrow H^{*}$, so that we can identify $(G / H)^{*}$ with the kernel of the restriction map res: $G^{*} \rightarrow H^{*}$. Moreover, we have res $\Delta(G)=\Delta(H)$. Our first result, then, exhibits the subspaces $(G / A)^{*}$ and $(G / B)^{*}$ in terms of the polyhedron $\Delta(G)$. The following terminology will be convenient: We say that the vector subspace $V \leqslant \mathbf{R}^{n}$ is an affine cartesian factor of the polyhedron $\Delta \subseteq \mathbf{R}^{n}$ if $\Delta=$ $\pi^{-1}(\pi(\Delta))$, where $\pi$ is the canonical projection $\mathbf{R}^{n} \rightarrow \mathbf{R}^{n} / V$ (so that $\Delta \cong V \times \pi(\Delta)$ ). Every polyhedron has a unique maximal affine cartesian factor.

Theorem A. If $A \leqslant B \leqslant G$ as above, then $(G / B)^{*} \subseteq \Delta(G) \subseteq(G / A)^{*}$. In fact we have

(a) $(G / A)^{*}$ is the subspace spanned by $\Delta(G)$,

(b) $(G / B)^{*}$ is the maximal affine cartesian factor of $\Delta(G)$.

By the Bergman carrier $\mathfrak{S}(\Delta(G))$ of $\Delta(G)$ we mean the uniquely determined (finite) set of subspaces $X \leqslant G^{*}$ such that the union $\bigcup\{X \mid X \in \subseteq(\Delta(G))\}$ contains $\Delta(G)$ and is minimal with respect to that property. By [2, Theorem 1.1], the dimension of each $X \in \mathfrak{S}(\Delta(G))$ is equal to the transcendence degree $m$ of $k(G)$ over $k$. We stress that nontriviality of $A$ and $G / B$ above should be considered as a degenerate situation. In the nondegenerate, "generic", case the polyhedron $\Delta(G)$ has a certain rigidity property which is the main result of this paper.

THEOREM B. If $A$ and $G / B$ are finite, then $G^{*}$ is spanned by the one-dimensional intersections $X_{1} \cap X_{2} \cap \cdots \cap X_{m}$ of spaces $X_{i}$ in the Bergman carrier $\mathfrak{E}(\Delta(G))$.

In the general case, Theorem $\mathrm{B}$ applies, of course, to the polyhedron $\Delta(B)=$ $\Delta(B / A)$ in $(B / A)^{*}$.

1.3. As an immediate consequence we obtain a new and more conceptual proof of Roseblade's key result [4, Theorem D]. This application is in the spirit of, but much stronger than, Bergman's proof of Zalesskii's conjecture [1].

Let $\Gamma$ be the group of all automorphisms of $k(G)$ over $k$ stabilizing $G$. Then both $A$ and $B$ are $\Gamma$ invariant so that $\Gamma$ acts on $A, B / A$ and $G / B$. The action on $A$ and $G / B$ is easy to understand: the automorphism group induced on $A$ is a subgroup of the Galois group of $k(A)$ over $k$ and hence is finite; and the automorphism group induced on $G / B$ is the full group $\mathrm{GL}_{r}(\mathrm{Z}), r=\operatorname{rk}(G / B)$. Thus the interesting part is the action on $B / A$.

The action of $\Gamma$ on $B$ induces an action on its character group $B^{*}$. Since $\Gamma$ permutes the valuations on $K$ over $k$, the subset $\Delta(B) \subseteq B^{*}$ is invariant under this action. Hence $\Gamma$ permutes the subspaces of the Bergman carrier $\mathfrak{S}(\Delta(B))$. Bergman knew that if $A \neq B$, then $\subseteq(\Delta(B))$ is nonempty and consists of proper subspaces of $G^{*}$, whence his conclusion that $\Gamma$ contains a subgroup of finite index stabilizing a proper subspace of $G^{*}$ and therefore a subgroup of infinite index in $G$. But now, with Theorem B available, we know that $\Gamma$ permutes a set of one-dimensional subspaces of $B^{*}$ spanning $(B / A)^{*}$. Hence $\Gamma$ contains a subgroup of finite index fixing $(B / A)^{*}$, and therefore $B / A$, pointwise. In other words we have

COROLlaRY. The automorphism group induced by $\Gamma$ on $B / A$ is finite. 
This is the core of the Brewster-Roseblade result [4, Theorem D]. Their full result, asserting that, in fact, the automorphism group induced by $\Gamma$ on $B$ is finite, can be deduced from the Corollary by an elementary argument which we sketch in the Appendix.

We observe that in the definition of $\Delta(G), G$ could be a torsion free subgroup in $K^{\times}$of finite rank, in which case $\Delta(G)$ coincides with $\Delta\left(G_{1}\right)$ for any finitely generated subgroup $G_{1}$ of maximum rank in $G$. The Corollary holds as before.

1.4. The proof of Theorem $A$ is given in $\S 2$. As to Theorem $B$, the only ingredients in its proof, apart from Theorem $\mathrm{A}$, are two geometric properties of $\Delta(G)$ which we established in [2]: homogeneity and concavity. $\$ \S 3,4$ and 5 contain a purely elementary-geometric analysis of these two conditions leading eventually to the rigidity result.

Finally, although we have followed Bergman in assuming that $k$ is a field, similar results hold in the more general case of $k$ being a Dedekind domain; we briefly sketch the necessary changes in $§ 6$.

The second author thanks the Deutsche Forschungsgemeinschaft for its financial support and the University of Frankfurt for its hospitality during the preparation of this paper.

\section{Proof of Theorem A.}

2.1. Let us first substantiate the remark we made immediately after stating Theorem A: that nontriviality of $G / B$ is to be considered a degenerate situation. As above let $K$ be a field, $k \subseteq K$ a subfield and $G \leqslant K$ a finitely generated multiplicative subgroup of $K$. Let $k G$ denote the group algebra of $G, k[G]$ the subalgebra of $K$ generated by $G$ over $k$, and $I$ the kernel of the canonical projection $k G \rightarrow k[G]$.

Proposition 2.1. If $H \leqslant G$ is a subgroup with torsion free factor $G / H$, then the following statements are equivalent:

(i) $I$ is controlled by $H$, that is, $I=(I \cap k H) k G$.

(ii) $k[G]$ is induced from $k[H]$, that is, the canonical map $k H \rightarrow k[H]$ induces an isomorphism $k[G] \cong k[H] \otimes_{k H} k G$.

(iii) $\operatorname{tr} \operatorname{deg}_{k(H)} k(G)=\operatorname{rk}(G / H)$.

(iv) $\Delta(G)=\operatorname{res}^{-1} \Delta(H)$.

Proof. (i) $\Leftrightarrow$ (ii) Tensoring the short exact sequence $I \cap k H \mapsto k H \rightarrow k[H]$ with $k G$ over $k H$ yields the short exact sequence

$$
(I \cap k H) k G \nrightarrow k G \rightarrow k[H] \otimes_{k H} k G,
$$

whence the assertion.

(ii) $\Leftrightarrow$ (iii) Let $X$ be a complement of $H$ in $G$. Then $k G \cong k H \otimes_{k} k X$, whence

$$
k[H] \otimes_{k H} k G \cong k[H] \otimes_{k} k X .
$$

This is just the Laurent polynomial ring of transcendence degree, over $k[H]$, equal to rk $X$, and so, in particular, a domain. Hence the canonical homomorphism $k[H] \otimes_{k H} k G \mapsto k[G]$ is an isomorphism if and only if the transcendence degrees (over $k[H]$, say) of the two domains are the same. 
(iii) $\Rightarrow$ (iv) Let $X$ be a complement of $H$ in $G$ and $\chi=\left(\chi_{H}, \chi_{X}\right) \in G^{*}$ with $\chi_{H} \in \Delta(H)$ and $\chi_{X} \in X^{*}$. Let $v: k(H)^{\times} \rightarrow \mathbf{R}$ be a valuation on $k(H)$ inducing $\chi_{H}$. Any basis of $X$ generates $k(G)$ over $k(H)$ and so must be algebraically independent. Hence $k(G)$ is just the field of fractions of the group ring $k(H) X$ and $v$ can be extended to a valuation $w: k(G)^{\times} \rightarrow \mathbf{R}$ inducing $\chi_{X}$. This shows that $\chi=\left(\chi_{H}, \chi_{X}\right) \in \Delta(G)$, as asserted.

(iv) $\Rightarrow$ (iii) By [2, Theorem 1.1] the dimension of $\Delta(G)$ coincides with the transcendence degree of $k(G)$ over $k$ and similarly for $\Delta(H)$. Hence $\operatorname{rk}(G / H)=$ $\operatorname{dim} \Delta(G)-\operatorname{dim} \Delta(H)=\operatorname{tr} \operatorname{deg}_{k(H)} k(G)$, as asserted.

2.2. The proof of Theorem A is now easily completed. The elements $x \in G$ which

are algebraic over $k$ are characterized by the property that $v(x)=0$ for all valuations on $K$ over $k$-in other words, by the property that $\Delta(G) \subseteq(G / \operatorname{gp}(x))^{*}$, where $\operatorname{gp}(x)$ stands for the subgroup generated by $x$. This shows that $(G / A)^{*}$ is the intersection of all rational hyperspaces of $\mathbf{R}^{n}$ containing $\Delta(G)$-a subspace of $\mathbf{R}^{n}$ is said to be rational if it is induced by a subspace of $Q^{n}$. But by [2, Theorem 1.1] we know that $\Delta(G)$ is a rational polyhedron, and so the intersection of all rational subspaces containing $\Delta(G)$ is, in fact, the subspace spanned by $\Delta(G)$. This proves assertion (a). As to (b), note that if $H$ is a subgroup of $G$, then $\Delta(G)$ is the cartesian product of $(G / H)^{*}$ and $\Delta(H)$ if and only if $\Delta(G)=\operatorname{res}^{-1} \Delta(H)$, and so, in view of Proposition 2.1, $(G / B)^{*}$ is the maximal rational subspace of $G^{*}$ with $\Delta(G)=$ $\operatorname{res}^{-1} \Delta(H)$. Since $\Delta(G)$ is a rational polyhedron the assertion follows.

\section{Polyhedrons.}

3.1. We have to recall some affine geometric notation from [2]. A subset $S$ of the affine space $\mathbf{R}^{n}$ is said to be a convex polyhedron if it can be written as the intersection of finitely many closed affine half spaces in $\mathbf{R}^{n}$. The dimension of the convex polyhedron $S$ is defined to be the dimension of the affine subspace of $\mathbf{R}^{n}$ spanned by $S$ and denoted $\operatorname{dim} S$. A subset $\Delta \subseteq \mathbf{R}^{n}$ is said to be a polyhedron if $\Delta$ can be written as the union

$$
\Delta=C_{1} \cup C_{2} \cup \cdots \cup C_{s}
$$

of a finite number of convex polyhedrons $C_{i}$. The dimension of $\Delta$, denoted $\operatorname{dim} \Delta$, is the maximum number $\operatorname{dim} C_{i}$ as $1 \leqslant i \leqslant s$. The polyhedron $\Delta$ is said to be homogenous if the decomposition (3.1) can be chosen such that $\operatorname{dim} \Delta=\operatorname{dim} C_{i}$ for all $1 \leqslant i \leqslant s$.

In order to describe the local behaviour of a polyhedron $\Delta$ at a point $x \in \Delta$ it is convenient to introduce the local cone of $\Delta$ at $x$, denoted $\operatorname{LC}_{x}(\Delta)$. It consists of all points $y \in \mathbf{R}^{n}$ with the property that the segment $\{x+\rho(y-x) \mid 0 \leqslant \rho \leqslant \varepsilon\}$ joining $x$ and $x+\varepsilon(y-x)$ is contained in $\Delta$ for some $\varepsilon>0$. Note that $\operatorname{LC}_{x}(\Delta)$ is a polyhedron which contains and is homeomorphic to an open neighbourhood of $x$ in $\Delta$. A point $x \in \Delta$ is said to be regular if its local cone $\operatorname{LC}_{x}(\Delta)$ is an affine subspace of $\operatorname{dimension}=\operatorname{dim} \Delta=m$. A nonregular point is said to be singular. By reg $\Delta$ and sing $\Delta$ we denote the set of all regular and singular points of $\Delta$, respectively. By the essential part of $\Delta$, denoted ess $\Delta$, we mean the closure of reg $\Delta$ in $\mathbf{R}^{n}$. ess $\Delta$ could also be described as the union of all $m$-dimensional $C_{i}$ 's occurring in (3.1). Although 
this second description does not make it apparent that ess $\Delta$ is independent of the decomposition (3.1), it does show that this is a homogenous polyhedron of dimension $m$. A similar argument shows that the set $\mathfrak{S}(\Delta)$ of all affine subspaces of $\mathbf{R}^{n}$ which are the local cone of some regular point of $\Delta$ is finite. This is the carrier of $\Delta$. The union of all affine subspaces in $\mathfrak{S}(\Delta)$ contains $\Delta$ and is minimal with respect to this property.

3.2. Let us consider the special case of an $n$-dimensional polyhedron in $\mathbf{R}^{n}$ in somewhat more detail. We prove

LEMMA 3.1. If $\Delta$ is an n-dimensional homogenous polyhedron in $\mathbf{R}^{n}$, but not equal to $\mathbf{R}^{n}$, then sing $\Delta$ is an $(n-1)$-dimensional homogenous polyhedron.

Proof. As $\Delta$ is a finite union of finite intersections of closed half spaces, the set theoretic complement $\Delta^{c}$ is a finite union of finite intersections of open half spaces. But the intersection of finitely many open half spaces in $\mathbf{R}^{n}$ is either empty or $n$-dimensional, whence the closure $\overline{\Delta^{c}}$ is an $n$-dimensional homogenous polyhedron. A point $x \in \Delta$ is singular if and only if $\operatorname{LC}_{x}(\Delta) \neq \mathbf{R}^{n}$ which means that every neighbourhood of $x$ in $\mathbf{R}^{n}$ contains points not in $\Delta$. In other words we have $\operatorname{sing} \Delta=\Delta \cap \overline{\Delta^{c}}$, and thus, in particular, $\operatorname{sing} \Delta$ is a polyhedron. Clearly, $\operatorname{dim}(\operatorname{sing} \Delta)$ $\leqslant n-1$. On the other hand $\mathbf{R}^{n}$ is the disjoint union of $\Delta^{c}$, reg $\Delta$ and $\operatorname{sing} \Delta$, and both reg $\Delta$ and $\Delta^{c}$ are nonempty and open in $\mathbf{R}^{n}$. Hence the complement of sing $\Delta$ in $\mathbf{R}^{n}$ is not connected and so $\operatorname{dim}(\operatorname{sing} \Delta) \geqslant n-1$. Finally, let $x \in \operatorname{sing} \Delta$, and let $U$ be an open ball in $\mathbf{R}^{n}$ with centre $x$. As $\Delta$ is homogenous, the set reg $\Delta$ of regular points is dense in $\Delta$ and so $U \cap \operatorname{reg} \Delta \neq \varnothing$. The argument above shows that, in fact, $\operatorname{dim}(U \cap \operatorname{sing} \Delta)=n-1$. Hence the regular points of $\operatorname{sing} \Delta$ are dense in $\operatorname{sing} \Delta$, which amounts to saying that $\operatorname{sing} \Delta$ is homogenous.

3.3. A polyhedron $\Delta$ has, of course, many decompositions of the form (3.1). For later applications, however, it will be crucial that there is a canonical one in favourable cases. Let us say that the union (3.1) is a convex cell decomposition of $\Delta$, if the intersection $C_{i} \cap C_{j}$ is empty or a common face of both $C_{i}$ and $C_{j}$, for all $i \neq j$. Unfortunately not every polyhedron has a canonical convex cell decomposition (try, e.g., the union of a plane $P$ and a line $L$ intersecting $P$ in a single point). But we can prove

LEMma 3.2. Every n-dimensional homogenous polyhedron $\Delta \subseteq \mathbf{R}^{n}$ has a canonical convex cell decomposition $\Delta=\cup_{i=1}^{r} C_{i}$, where each $C_{i}$ is an n-dimensional convex polyhedron and

$$
\mathfrak{S}(\operatorname{sing} \Delta)=\bigcup_{i=1}^{r} \mathbb{E}\left(\operatorname{sing} C_{i}\right) \text {. }
$$

Note that $\sqrt{5}\left(\operatorname{sing} C_{i}\right)$ is, of course, just the set of all affine subspaces spanned by the $(n-1)$-dimensional faces of the convex polyhedron $C_{i}$. Any face of $C_{i}$ contained in sing $\Delta$ will be called a face of $\Delta$. The faces of $\Delta$ form a convex cell decomposition of the homogenous polyhedron sing $\Delta$. 
Proof (of Lemma 3.2). $\sqrt{5}$ (sing $\Delta$ ) is a finite set of affine hyperspaces of $\mathbf{R}^{n}$ and so defines a convex cell decomposition of the affine space $\mathbf{R}^{n}$,

$$
\mathbf{R}^{n}=\bigcup_{j=1}^{s} D_{j},
$$

where $D_{1}, D_{2}, \ldots, D_{s}$ are $n$-dimensional convex polyhedrons. Let $\stackrel{\circ}{D}_{j}$ denote the interior of the convex cell $D_{j}$. Clearly, $\stackrel{\circ}{D}_{j}$ has empty intersection with sing $\Delta$, whence

$$
\stackrel{\circ}{D}_{j}=\left(\stackrel{\circ}{D}_{j} \cap \operatorname{reg} \Delta\right) \cup\left(\stackrel{\circ}{D}_{j} \cap \Delta^{c}\right) .
$$

Thus $\stackrel{\circ}{D}_{j}$ is the union of two disjoint open sets. As $\stackrel{D}{D}_{j}$ is connected, one of these must be empty, that is, either $\stackrel{\circ}{D}_{j} \subseteq \Delta$ or $\stackrel{\circ}{D}_{j} \subseteq \Delta^{c}$. It follows that $\Delta$ is the union of all convex cells $D_{j}$ contained in $\Delta$, whence the lemma.

COROllaRY 3.3. Let $\Delta \subseteq \mathbf{R}^{n}$ be an $n$-dimensional homogenous polyhedron and $L \subseteq \mathbf{R}^{n}$ a line which intersects but is not contained in $\Delta$. Then the carrier $\subseteq(\operatorname{sing} \Delta)$ of sing $\Delta$ contains a hyperspace $X$ which intersects $L$ in a single point.

Proof. By Lemma 3.2 we may assume that $\Delta$ is convex. Then we proceed by induction on $n$. As $\Delta \cap L$ is neither empty nor all of $L$, there is a point $x \in L \cap \operatorname{sing} \Delta$. Hence there is an $(n-1)$-dimensional face $F$ of $\Delta$ with $x \in L \cap F$. Let $Y$ be the affine subspace spanned by $F$. Then $Y \in \mathcal{C}(\operatorname{sing} \Delta)$, and if $L \cap Y$ is the singleton set $\{x\}$, we put $X=Y$ and are done. Otherwise, $L \subseteq Y$ and, by induction, there is an $(n-2)$-dimensional face $F_{1}$ of $F$ such that the intersection of $L$ with the subspace $Z_{1}$ spanned by $F_{1}$ is a singleton. But $F_{1}$ is the face of exactly two $(n-1)$-dimensional faces, one of which is $F$ (see e.g. [3, Satz 5.2]). The subspace $X$ spanned by the other one cannot contain $L$, whence the corollary.

4. The effect of total concavity.

4.1. Let $\Delta \subseteq \mathbf{R}^{n}$ be a polyhedron. Recall from [2] that $\Delta$ is said to be concave at a point $x \in \Delta$ if the convex hull of the local cone $\operatorname{LC}_{x}(\Delta)$ is an affine subspace of $\mathbf{R}^{n}$. $\Delta$ is totally concave if it is concave at all points $x \in \Delta$.

LEMma 4.1. If $\Delta$ is a homogenous $n$-dimensional totally concave polyhedron in $\mathbf{R}^{n}$, then $\Delta=\mathbf{R}^{n}$.

Proof. According to the proof of Lemma 3.2 the affine space $\mathbf{R}^{n}$ has a (finite) convex cell decomposition $\mathbf{R}^{n}=C_{1} \cup C_{2} \cup \cdots \cup C_{r}$ with each $C_{i} n$-dimensional, such that $\Delta$ is the union of a subset of the $C_{i}$ 's. If $\Delta \neq \mathbf{R}^{n}$, then there is a pair of indices $i, j$ such that $C_{i}$ and $C_{j}$ have a common $(n-1)$-dimensional face $F, C_{i} \subseteq \Delta$ and $C_{j} \nsubseteq \Delta$. Then $\Delta$ is not concave at the regular points of $F$.

4.2. Let $\Delta \subseteq \mathbf{R}^{n}$ be an arbitrary homogenous $m$-dimensional polyhedron. Then the set reg $\Delta$ of all regular points of $\Delta$ is dense in $\Delta$, hence every point $x \in \Delta$ is contained in the essential part of $\Delta \cap X$ for some $X \in \mathfrak{S}(\Delta)$; that is, one has the canonical decomposition

$$
\Delta=\bigcup_{X \in \mathbb{S}(\Delta)} \operatorname{ess}(\Delta \cap X)
$$


Note that each of the polyhedrons $\operatorname{ess}(\Delta \cap X)$ is $m$-dimensional, homogenous and contained in some $X=\mathbf{R}^{m}$, whence has a canonical convex cell decomposition by Lemma 3.2.

Proposition 4.2. Let $\Delta \subseteq \mathbf{R}^{n}$ be a homogenous polyhedron and $X \in \mathfrak{\subseteq}(\Delta)$. If $\Delta$ is totally concave, then every space $Z$ in the carrier $\mathbb{C}(\operatorname{sing}(\operatorname{ess}(\Delta \cap X)))$ is the intersection $Z=X \cap Y$ of $X$ with some other $Y \in \mathbb{S}(\Delta)$.

Proof. $Z$ is the local cone of some regular point $z$ of the polyhedron $\operatorname{sing}(\operatorname{ess}(\Delta \cap X))$. If $z \notin \operatorname{ess}(\Delta \cap Y)$ for all $Y \in \mathbb{C}(\Delta)$ other than $X$, then there is an open neighbourhood $U$ of $z$ in $\mathbf{R}^{n}$ with

$$
\operatorname{ess}(\Delta \cap Y) \cap U=\varnothing, \text { all } X \neq Y \in \mathbb{E}(\Delta) \text {. }
$$

Hence $\Delta \cap U=\operatorname{ess}(\Delta \cap X) \cap U$ by (4.1). This shows that the behaviour of $\Delta$ in a neighbourhood of $z$ is given by $\operatorname{ess}(\Delta \cap X)$. From that we infer that the local cones of $\Delta$ and $\operatorname{ess}(\Delta \cap X)$ at $z$ coincide and are totally concave. But since $\operatorname{LC}_{x}(\operatorname{ess}(\Delta \cap X))$ is homogenous and of dimension $=\operatorname{dim} X$, Lemma 4.1 applies and $\mathrm{LC}_{z}(\operatorname{ess}(\Delta \cap X))=X$, contradicting the assumption that $z$ be a singular point of $\operatorname{ess}(\Delta \cap X)$. This shows that there is some $Y \in \subseteq(\Delta), Y \neq X$, with $z \in X \cap Y$. The same arguments apply for all points in a neighbourhood of $z$ in $\operatorname{sing}(\operatorname{ess}(\Delta \cap X))$; and so, as $\mathfrak{S}(\Delta)$ is finite, one of the subspaces $Y \in \mathfrak{C}(\Delta), Y \neq X$, must contain such a neighbourhood and hence $Z$. Since $\operatorname{dim} Z=\operatorname{dim}(\operatorname{sing}(\operatorname{ess}(\Delta \cap X)))=\operatorname{dim} \Delta-1$ $\geqslant \operatorname{dim}(X \cap Y)$, we have $Z=X \cap Y$, as asserted.

4.3. As a consequence of Proposition 4.2 we obtain that homogenous totally concave polyhedrons behave somewhat similarly to the set of all singular points of an $n$-dimensional convex polyhedron in $\mathbf{R}^{n}$ (compare Corollary 3.3).

Proposition 4.3. Let $\Delta$ be a homogenous and totally concave polyhedron in $\mathbf{R}^{n}$, and $L \subseteq \mathbf{R}^{n}$ a line which intersects but is not contained in $\Delta$. Then there is some $X \in \mathfrak{S}(\Delta)$ which intersects $L$ in a single point.

Proof. As $\Delta$ is homogenous, there is some $X \in \mathbb{E}(\Delta)$ such that $L$ intersects but is not contained in ess $(\Delta \cap X)$. If $L \subseteq X$, then we are done; otherwise Corollary 3.3 applies for $\operatorname{ess}(\Delta \cap X)$ and yields $Z \in \mathbb{C}(\operatorname{sing}(\operatorname{ess}(\Delta \cap X)))$ such that $Z \cap L$ is a singleton. By Proposition 4.2 there is $Y \in \mathfrak{E}(\Delta)$ with $X \cap Y=Z$ and $Y$ cannot contain $L$ since $X \neq Y$. Hence $Y \cap L$ consists of a single point.

4.4. Define the vector carrier $\mathfrak{B} \mathfrak{C}(\Delta)$ to be the set of vector subspaces $V \leqslant \mathbf{R}^{n}$ such that some affine translate of $V$ lies in $\sqrt{5}(\Delta)$. This, of course, is equal to $\sqrt{5}(\Delta)$ in case $\Delta$ is a cone.

COROllary 4.4. Let $\Delta \leqslant \mathbf{R}^{n}$ be a polyhedron which is homogeneous and totally concave. Then the intersection $V$ of all spaces in $\mathfrak{B} \mathfrak{S}(\Delta)$ is an affine cartesian factor of $\Delta$.

Proof. Let $\pi$ be the projection $\pi: \mathbf{R}^{n} \rightarrow \mathbf{R}^{n} / V$. We must show that $\pi^{-1}(\pi(\Delta))=\Delta$ or, equivalently, that $\Delta+V=\Delta$. Now $\Delta+V=\Delta$ if and only if $z+\mathbf{R} v \subseteq \Delta$ for all $z \in \Delta, v \in V$; that is, if and only if every line parallel to $V$ which meets $\Delta$ lies 
wholly in $\Delta$. But a line is parallel to $V$ if and only if it is parallel to each $X \in \mathfrak{B} \subseteq(\Delta)$ or, equivalently, each $X \in \mathfrak{S}(\Delta)$. Thus Proposition 4.3 implies that $V$ is an affine cartesian factor of $\Delta$.

\section{Proof of Theorem B.}

5.1. Let $K$ be a field, $k \subseteq K$ a subfield and $G \leqslant K^{\times}$a finitely generated multiplicative subgroup of $K$. Since positive multiples of valuations over $k$ are again valuations over $k$, the polyhedron $\Delta(G) \subseteq G^{*}$ is a cone. Moreover, we have shown in [2] that $\Delta(G)$ is homogenous and totally concave.

As to homogeneity, we can do better than this. If $H \leqslant G$ is a subgroup, then the restriction map res: $G^{*} \rightarrow H^{*}$ is $\mathbf{R}$-linear and maps $\Delta(G)$ onto $\Delta(H)$. This shows that not only $\Delta(G)$ is homogenous but also every image of $\Delta(G)$ under linear maps induced by embeddings of subgroups. We leave it to the reader to extend this observation to arbitrary linear maps, that is, to prove

THEOREM 5.1. The polyhedron $\Delta(G) \subseteq G^{*}=\mathbf{R}^{n}$ has the property that all its images under $\mathbf{R}$-linear maps $\pi: \mathbf{R}^{n} \rightarrow \mathbf{R}^{n}$ are homogenous.

We shall really need the result only for the case when $\pi$ is induced by the embedding of a subgroup of corank 1 .

5.2. It is now clear that Theorem B is an immediate consequence of Theorem $A$ and the following purely geometric result. (Observing that, as $\Delta$ is a cone, the carrier and vector carrier of $\Delta$ coincide.)

Proposition 5.2. Let $\Delta \subseteq \mathbf{R}^{n}$ be a polyhedron with the following properties.

(i) $\Delta$ is totally concave.

(ii) All images of $\Delta$ under linear maps $\mathbf{R}^{n} \rightarrow \mathbf{R}^{m}$ are homogeneous.

(iii) $\Delta$ has no nontrivial affine cartesian factor.

Then the subspace $\mathbf{R}^{n}$ spanned by $\mathfrak{B} \mathfrak{S}(\Delta)$ is spanned by one-dimensional subspaces of the form $X_{1} \cap X_{2} \cap \cdots \cap X_{r}, X_{i} \in \mathfrak{B} \mathfrak{S}(\Delta)$.

5.3. The proof of Proposition 5.2 relies on

LEMMA 5.3. Let $V$ be an $n$-dimensional vector space over any field $K$ and $\sqrt{5}$ a finite family of $(n-1)$-dimensional subspaces. If $\mathbb{5}$ has the property that it contains complements to every one-dimensional subspace $L \leqslant V$ in $V$, then $V$ is spanned by the one-dimensional subspaces of the form $X_{1} \cap X_{2} \cap \cdots \cap X_{n-1}, X_{i} \in \mathbb{C}$.

Proof (By INDUCTION ON $n$ ). If $n=2$, the spaces in 5 are one-dimensional themselves and there are at least two different ones. So let $n>2$, pick $X \in \mathbb{C}$, and consider

$$
\mathfrak{S}_{X}=\{X \cap Y \mid X \neq Y \in \mathbb{E}\}
$$

which is a finite family of $(n-2)$-dimensional subspaces of $X$. For every onedimensional subspace $L \leqslant X$ there is $Y \in \mathbb{C}$ with $V=L \oplus Y$. Note $L \nless Y$ so that $Y \neq X$, whence $X=L \oplus(X \cap Y)$. By induction $X$ is spanned by one-dimensional subspaces of the form $X \cap Y_{i} \cap \cdots \cap Y_{n-2}, Y_{i} \in \mathbb{E}$. This holds for all $X \in \mathbb{E}$ and $\checkmark$ contains more than one space. 
5.4. Proof of Proposition 5.2. Let $X \in \mathfrak{B} \subseteq(\Delta), L \leqslant X$ a one-dimensional subspace and $\pi: \mathbf{R}^{n} \rightarrow \mathbf{R}^{n-1}$ a linear map with kernel $L$. Let $m=\operatorname{dim} \Delta$. If $\operatorname{dim} \pi(\Delta)=m-1$, then $L \subseteq Y$ for every $Y$ in the carrier $\mathfrak{B} \subseteq(\Delta)$. But that would mean, by Corollary 4.4 , that $L$ is a cartesian factor of $\Delta$. Therefore $\operatorname{dim} \pi(\Delta)=m$. But certainly the dimension of $\pi(X)$ is equal to $m-1$, and so, as $\pi(\Delta)$ is homogenous, $\pi(X)$ must be contained in $\pi(Y)$ for some $Y \in \mathfrak{B} \mathfrak{S}(\Delta), X \neq Y$. In other words $X \subseteq Y+L$ and hence $X=(Y \cap X) \oplus L$. This shows that the family

$$
\mathbb{E}_{X}=\{X \cap Y \mid X \neq Y \in \mathfrak{B} \subseteq(\Delta)\}
$$

of subspaces of $X$ satisfies the assumption of Lemma 5.3. Hence $X$ is spanned by one-dimensional intersections of subspaces in the carrier of $\Delta$. This holds for all $X \in \mathfrak{B} \subseteq(\Delta)$, whence the assertion.

This completes the proof of Proposition 5.2 and hence that of Theorem B.

6. Note on a generalization. The assumption that the valuations considered on the field $K$ are trivial when restricted to the base field $k$ is unnecessarily strong. One can take an arbitrary discrete valuation $v: k^{\times} \rightarrow \mathbf{Z}$ and consider the set $\Delta^{v}(G)$ of all characters of $G$ induced by a real valuation on $K$ extending $v$. Then $\Delta^{v}(G)$, although not necessarily a cone, is homogeneous and totally concave and does have the property in Proposition 5.1 (see [2]).

The proof of all the intermediate results in $\S \S 2-5$ now carry through for $\Delta^{v}(G)$ (as well as for the global sets $\Delta^{k / D}(G)$ for $D \subseteq k$ a Dedekind domain; see [2]) yielding appropriate versions of Theorems A and B. More precisely, in Theorem A, we must replace "the subspace spanned by $\Delta(G)$ " by "the subspace spanned by the vector carrier of $\Delta^{v}(G)$ " and in Theorem B we must replace " $\subseteq(\Delta(G))$ " by “ $\mathfrak{B} \subseteq\left(\Delta^{v}(G)\right)$ ".

However, it should be noticed that by [2, Theorem C1], $\Delta(G)=\Delta^{0}(G)$ is the local cone of $\Delta^{v}(G)$ at infinity, and so the vector carrier of $\Delta^{v}(G)$ contains the Bergman carrier of $\Delta^{0}(G)$. Moreover, it can be shown (by using Proposition 2.1 or a geometric argument involving total concavity) that the maximal affine cartesian factors of $\Delta^{0}(G)$ and $\Delta^{v}(G)$ coincide. Hence the rigidity result for $\Delta^{v}(G)$ is no stronger than the rigidity result for $\Delta^{0}(G)$ - and this is the reason why we restricted attention to the field case.

Appendix. We sketch the proof that our Corollary in the introduction implies the full result of Theorem D in [4]. We show that, in fact, the automorphism group induced by $\Gamma$ on $B$ is finite.

We have shown that the automorphism groups induced by $\Gamma$ on both $B / A$ and $A$ are finite. Thus it suffices to show that any automorphism $\rho$ which is trivial on both $B / A$ and $A$ has finite order.

Let $F \leqslant k(B)$ be the fixed field of $\rho$ and let $\bar{F}$ be its algebraic closure in $k(B)$. Then $|\bar{F}: F|$ is finite and so, after passing to a suitable power of $\rho$, we may assume that $\bar{F}=F$. We claim now that $k(B)=F$; we suppose not and derive a contradiction.

Let $k(B)=F\left(x_{1}, \ldots, x_{s}\right)$ with $\left\{x_{i}\right\}_{i=1}^{s} \subset B$ multiplicatively independent. By the definition of $B$ the $x_{i}$ cannot be algebraically independent over $F$; choose $x_{1}, \ldots, x_{t}$ 
so that $x_{1}, \ldots, x_{t-1}$ are algebraically independent over $F$ and $x_{t}$ is algebraic over $F\left(x_{1}, \ldots, x_{t-1}\right)$. Let $P\left(X_{1}, \ldots, X_{t}\right) \in F\left[X_{1}, \ldots, X_{t}\right]$ be the minimal polynomial of $x_{t}$ over $F\left[x_{1}, \ldots, x_{t-1}\right]$.

Since $\rho$ fixes both $B / A$ and $A$, we have $\rho\left(x_{i}\right)=x_{i} a_{i}$ with $a_{i} \in A \subseteq F$. Also, as $F$ is the fixed field of $\rho$ and is algebraically closed in $k(B)$, the $a_{i}$ are multiplicatively independent. Now, $P\left(x_{1} a_{1}, \ldots, x_{t} a_{t}\right)=0$ and so $P\left(X_{1} a_{1}, \ldots, X_{t} a_{t}\right)$ is a multiple, clearly a $F$-scalar multiple, of $P\left(X_{1}, \ldots, X_{t}\right)$. Comparing coefficients of monomials and recalling that the $a_{i}$ are multiplicatively independent, we observe that $P$ is a monomial in the $X_{i}$-an evident contradiction.

Thus $F=k(B)$ and so $\rho$ (or, in the original statement, some power of $\rho$ ) is trivial.

\section{REFERENCES}

1. G. M. Bergman, The logarithmic limit-set of an algebraic variety, Trans. Amer. Math. Soc. 157 (1971), 459-469.

2. R. Bieri and J. R. J. Groves, The geometry of the set of characters induced by valuations, J. Reine Angew. Math. 347 (1984), 168-195.

3. K. Leichtweiss, Konvexe Mengen, Hochschultext, Springer-Verlag, Berlin, Heidelberg and New York, 1980.

4. J. E. Roseblade, Prime ideals in group rings of polycyclic groups, Proc. London Math. Soc. (3) 36 (1978), 385-447.

Department of Mathematics, University of Frankfurt, 6000 Frankfurt am Main, West (iERMANY

Department of Mathematics, University of Melbourne, Parkville, Victoria 3052, Australia 